\title{
Atom Probe Tomography and Correlative Microscopy: 3D Nanoscale Characterization of Metals, Minerals and Materials
}

\author{
ARUN DEVARAJ (i) ${ }^{1,3}$ and PHILIP EISENLOHR ${ }^{2,4}$ \\ 1.-Physical and Computational Sciences Directorate, Pacific Northwest National Laboratory, \\ Richland, WA, USA. 2.-Chemical Engineering and Materials Science, Michigan State University, \\ East Lansing, MI, USA. 3.—e-mail: arun.devaraj@pnnl.gov. 4.—e-mail: eisenlohr@egr.msu.edu
}

Atom probe tomography (APT) is a three-dimensional (3D) nanoscale compositional characterization method, now widely used for analyzing a variety of materials. The 3D nanoscale composition obtained using APT is complementary to the structural and chemical state information obtained using other methods such as transmission electron microscopy (TEM), x-ray diffraction, or x-ray absorption spectroscopy. The use of correlative microscopy, where the same specimen is analyzed using multiple characterization methods, has progressively increased in the materials science community over recent years.

This JOM special topic presents a collection of papers demonstrating recent progress in the utilization of APT along with correlative microscopy where the 3D nanoscale compositional information obtained using APT complements other structural or chemical state characterization results. This unique combination is shown to facilitate the understanding of phase transformation mechanisms, structure-property relationships of materials, grain boundary segregation, nanoscale precipitation, and quantifying the extent of solute segregation to defects and interfaces in materials to understanding degradation of materials due to corrosion.

A chronological review of developments in correlative microscopy between APT and other methods such as TEM imaging, scanning TEM (STEM) tomography, nanobeam diffraction, electron backscatter diffraction (EBSD), scanning electron microscopy (SEM) imaging, and computational simulations are provided in the review article by $\mathrm{P}$. Kolli. This paper provides an introduction to the various topic areas covered in this special issue.

Arun Deravaj and Philip Eisenlohr are the JOM advisors for the Advanced Characterization, Testing, and Simulation Committee of the TMS Structural Materials Division, and guest editors for the topic 3D Nanoscale Characterization of Metals, Minerals, and Materials in this issue.
APT and correlative microscopy is very impactful in understanding solute segregation to deformationinduced defects such as dislocations and stacking faults. The paper by S. Makineni and coworkers provides a comprehensive review of three different approaches for analyzing solute segregation to defects and interfaces in deformed metallic alloys. It is noted that specimen preparation is one of the most critical steps that directly determine the outcome of the correlative microscopy approaches. Therefore, the paper stresses the careful identification of regions using detailed SEM-based electron channeling contrast imaging or TEM imaging followed by protection of the region of interest by $\mathrm{Pt}$ deposition prior to sample extraction and preparation of APT needles.

APT has also made significant contributions recently to the understanding of material degradation due to corrosion. The review article by K. Eder provides a broad overview of the usage of APT and correlative microscopy for understanding the structural and compositional changes of metallic alloys due to corrosion. Examples for the usage of TEM as well as transmission Kikuchi diffraction methods correlated with APT are provided in their work.

The paper by A. Devaraj et al. highlights the unique benefit of APT correlated with SEM imaging and EBSD to help quantify carbon concentration and other solute partitioning between different phases in a commercial quenched and partitioned steel. Quantitative analysis of light elements is one of the biggest advantages of APT compared to other microstructural analysis methods such as electron microscopy or energy dispersive spectroscopy.

Highly deformed metallic alloys have complicated microstructures due to a high concentration of defects. Studying the compositional and structural details of highly deformed metallic alloys in a quantitative manner at sub-nanometer spatial resolution is a challenging task for most of the established characterization methods. The work by S. 
Medghalchi et al. demonstrates that, by using methods such as x-ray diffraction and TEM, various regions of deformed metallic components can be identified for detailed compositional analysis by using APT. Thus, use of APT along with XRD and TEM can reveal the effect of deformation-induced local annealing on solute redistribution in the microstructure of industrially relevant components, for instance, gear teeth subjected to cyclic loading.

APT has made crucial contributions towards understanding the phase transformations in many metallic alloys. The publication by M.D. Giovanni et al. is an example of using APT to obtain additional quantitative information on Y segregation to interfaces in Al-Si alloys. The publication by T. Alam et al. highlights the unique advantage of APT in analyzing core-shell precipitates of less than $10 \mathrm{~nm}$ in size in complex high entropy alloys.

Finally, the instrumentation of APT has been under continuous development to increase, for instance, the detection efficiency by improving detectors or the mass-to-charge spectra resolution by incorporation of a reflectron, to name a few. More recently, a concurrent development to reduce the cost-of-ownership and provide easier to use functionalities has also been initiated. As a result, a new commercial APT system was recently developed with a modified local electrode geometry and a 532$\mathrm{nm}$ laser. The publication by D.J. Larson and coworkers provides the first detailed description of this new APT system as well as characterization results for a number of materials. Usage of correlative transmission Kikuchi diffraction with this new APT system for analyzing specific microstructural features in materials is also provided in their manuscript.

The following list summarizes the papers being published under the topic of "Atom Probe Tomography and Correlative Microscopy: 3D Nanoscale Characterization of Metals, Minerals, and Materials". To download any of the papers, follow the URL http://link.springer.com/journal/11837/70/9/page/1 to the table of contents page for the September 2018 issue (vol. 70, no. 9).
- "Atom Probe Tomography: A Review of Correlative Analysis of Interfaces and Precipitates in Metals and Alloys" by R. Prakash Kolli.

- "Correlative Microscopy-Novel Methods and Their Applications to Explore 3D Chemistry and Structure of Nanoscale Lattice Defects: A Case Study in Superalloys" by S.K. Makineni, M. Lenz, P. Kontis, Z. Li, A. Kumar, P.J. Felfer, S. Neumeier, M. Herbig, E. Spiecker, D. Raabe, and B. Gault.

- "Nanoscale Analysis of Corrosion Products: A Review of the Application of Atom Probe and Complementary Microscopy Techniques" by Katja Eder, Ingrid McCarroll, Alexandre La Fontaine, and Julie M. Cairney.

- "Nanoscale Solute Partitioning and Carbide Precipitation in a Multiphase Trip Steel Analyzed by Atom Probe Tomography" by Arun Devaraj, Zeren Xu, Fadi Abu-Farha, Xin Sun, and Louis G. Hector Jr.

- "Multiscale Characterization of Microstructure in Near-Surface Regions of a $16 \mathrm{MnCr} 5$ Gear Wheel After Cyclic Loading" by Setareh Medghalchi Vahid Jamebozorgi, Arjun Bala Krishnan, Smobin Vincent, Steffen Salomon, Alireza Parsa, Janine Pfetzing, Aleksander Kostka, Yujiao Li, and Tong Li.

- "3D Atom Probe Tomography Study on Segregation of Yttrium in Modified Al-Si Alloys" by Mario De Giovanni, Talukder Alam, Raj Banerjee, and Prakash Srirangam.

- "Detailed Investigation of Core-Shell Precipitates in a Cu-Containing High Entropy Alloy" by T. Alam, B. Gwalani, G. Viswanathan, H. Fraser, and R. Banerjee.

- "Microstructural Investigations in Metals Using Atom Probe Tomography with a Novel Specimen-Electrode Geometry" by David J. Larson, Robert M. Ulfig, Dan R. Lenz, Joseph H. Bunton, Jeff D. Shepard, Timothy R. Payne, Katherine P. Rice, Yimeng Chen, Ty J. Prosa, Dan J. Rauls, Thomas F. Kelly, Niyanth Sridharan, and Suresh Babu. 